\title{
Liquid Phase Nitration of Benzene Catalyzed by a Novel Salt of Molybdovanadophosphoric Heteropolyacid
}

\author{
Jianan Liu, ${ }^{a}$ Yanan Wang, ${ }^{a}$ Shuwen Gong, ${ }^{\odot *, a}$ Wenzeng Duan ${ }^{a}$ and Xianqiang Huang ${ }^{a}$ \\ ${ }^{a}$ Institution of Functional Organic Molecules and Materials, \\ School of Chemistry and Chemical Engineering, Liaocheng University, \\ 252000 Liaocheng, P. R. China
}

\begin{abstract}
A highly efficient and reusable catalyst QA-HPMV was successfully prepared by the reaction of quinoline-2-formic acid (QA) with molybdovanadophosphoric heteropolyacid $\left(\mathrm{H}_{4} \mathrm{PMo}_{11} \mathrm{VO}_{40}\right.$, HPMV) for the nitration of benzene. The physical and chemical properties of the sample was characterized by X-ray diffraction analysis (XRD), Fourier transform infrared spectroscopy (FTIR), UV-Vis and thermogravimetric analysis (TG). The characterization results showed that the formed QA-HPMV retained the Keggin structure of HPMV. As a catalyst for benzene nitration, QA-HPMV showed good catalytic performance and the yield of nitrobenzene was $82.5 \%$ under the optimized reaction conditions. As a heterogeneous catalyst, QA-HPMV can be easily recycled from the reaction medium by filtering and remained highly catalytic performance even after five runs of recycling.
\end{abstract}

Keywords: molybdovanadophosphoric heteropolyacid, solid acid, catalysis, benzene, liquid phase nitration

\section{Introduction}

Nitrobenzene is an important organic chemical material. It can be used as an intermediate for organic synthesis. In addition to being used as raw material to synthesize aniline, nitrobenzene can also be used to synthesize some dyes, spices, explosives and so on. ${ }^{1-5}$ The synthesis of nitrobenzene generally use the nitration of benzene and nitric acid, which can be divided into liquid phase nitration, gas-liquid phase nitration and gas phase nitration according to the physical state of the reaction material. ${ }^{2,6,7}$

The typical liquid phase nitration not only takes benzene and nitric acid as raw materials, but also sulfuric acid is used as catalyst. Although sulfuric acid has remarkable catalytic activity, it causes corrosion problems and is not environment-friendly. A large amount of waste acid is produced in the nitration process, which inevitably results in a high cost of recovery and generates environmental hazards. In order to overcome these shortages of sulfuric acid as catalyst, solid acid catalysts were developed for nitration of benzene. ${ }^{7,8}$

As an alternative to sulfuric acid, solid acid catalysts have attracted wide attention because of their advantages such as easy separation from products, convenience for

*e-mail: gshw76@163.com recycling, and environmental friendliness. ${ }^{6,8-10}$ In recent years, some solid acids such as zeolite, ${ }^{11,12} \mathrm{WO}_{3} / \mathrm{ZrO}_{2},{ }^{13}$ $\mathrm{Pt}-\mathrm{Cu} / \mathrm{TiO}_{2}{ }^{14}$ have been applied as catalyst in the nitration of benzene.

As a kind of environmentally friendly material, heteropolyacids with Keggin structure have been widely studied as catalyst for many reactions, including the catalytic synthesis of heterocyclic compounds, ${ }^{15,16}$ nitration reaction, ${ }^{3,17}$ oxidation reaction, ${ }^{18}$ and so on. For example, Romanelli et al. ${ }^{19}$ reported the two-step synthesis of 6-cyano-2,2-dimethyl-2- $H$-1-benzopyran with 3-methyl2-butenal as starting material and Keggin heteropoly acid $\mathrm{H}_{4} \mathrm{PMo}_{11} \mathrm{VO}_{40}\left(\mathrm{PMo}_{11} \mathrm{~V}\right)$ or $(\mathrm{PyH})_{3} \mathrm{HPMo}_{11} \mathrm{VO}_{40}$ $\left(\mathrm{Py}_{3} \mathrm{PMo}_{11} \mathrm{~V}\right)$ as catalyst, with a yield of $35 \%$. You et al. ${ }^{20}$ once reported that the supported HPW $\left(\mathrm{H}_{3} \mathrm{PW}_{12} \mathrm{O}_{40}\right) /$ MCM-41 has obvious catalytic performance in the vapor phase nitration of benzene and the 50\% HPW/MCM-41 catalyst can make benzene conversion rate of $73.4 \%$.

In this work, a novel heteropoly acid salt QA-HPMV was obtained by the reaction of quinoline-2-formic acid (QA) with molybdovanadophosphoric heteropolyacid $\left(\mathrm{H}_{4} \mathrm{PMo}_{11} \mathrm{VO}_{40}, \mathrm{HPMV}\right)$. It was found that QA-HPMV has good catalytic activity in the liquid phase nitration of benzene to nitrobenzene. In addition to the higher yield, the better advantage of QA-HPMV was that can be easily recovered and reused for many times. 


\section{Experimental}

\section{Catalyst preparation}

All the reagents were of analytical reagent (AR) grade and used as received without further purification. The synthesis of HPMV was based on the method reported in the literature. ${ }^{21} \mathrm{Na}_{2} \mathrm{HPO}_{4} \cdot 12 \mathrm{H}_{2} \mathrm{O}(0.005 \mathrm{~mol}$, Tianjin Damao Chemical Reagent Factory, Tianjin, China) was dissolved in deionized water at room temperature. $\mathrm{NaVO}_{3}(0.005 \mathrm{~mol}$, Aladdin Industrial Corporation, Shanghai, China) was dissolved in deionized water under boiling condition. After the two solutions were mixed together and cooled to room temperature, the mixed solution was acidified to red with concentrated sulfuric acid. Then, the aqueous solution containing $\mathrm{Na}_{2} \mathrm{MoO}_{4} \cdot 2 \mathrm{H}_{2} \mathrm{O}(0.055 \mathrm{~mol}$, Aladdin Industrial Corporation, Shanghai, China) was added with stirring. The sulfuric acid was added dropwise until the $\mathrm{pH}$ of the mixture was equal to 1 . When the mixture was cooled to room temperature, it was extracted with anhydrous ether and collected the intermediate layer. Finally, HPMV was obtained when the intermediate layer ether was removed and dried at $50{ }^{\circ} \mathrm{C}$ in oven.

QA-HPMV was obtained by the reaction of HPMV and quinoline-2-formic acid (QA) in aqueous solution. In a typical procedure, the QA $(0.004 \mathrm{~mol})$ aqueous solution was added dropwise to the HPMV (0.001 mol) aqueous solution with stirring at room temperature. Then, the resultant mixture was further stirred for $5 \mathrm{~h}$. Finally, the solid sample was obtained by filtration and dried at $80^{\circ} \mathrm{C}$, which was abbreviated as QA-HPMV and was evaluated directly as catalyst. At the same time, similar method was used to prepare other control samples by changing QA to nicotinic acid (NA) or dodecyl trimethyl ammonium bromide (DTAB), respectively. These obtained samples were named as NA-HPMV and DTAB-HPMV.

\section{Catalyst characterization}

The X-ray diffraction analysis (XRD) of the catalyst was carried out on the SmartLab $9 \mathrm{~kW}$ X-ray diffractometer (Rigaku, Tokyo, Japan) using Ni-filtered $\mathrm{Cu} \mathrm{K} \alpha$ radiation $(\lambda=0.15418 \mathrm{~nm})$ at voltage and current of $45 \mathrm{kV}$ and $200 \mathrm{~mA}$, respectively. The powder XRD pattern in the $2 \theta$ range of $5-80^{\circ}$ was recorded at speed of 20 degree $\mathrm{min}^{-1}$. The infrared spectrum (IR) analysis was performed on a Nicolet 6700 FT-TR spectrometer (Thermo Fisher Scientific, Waltham, USA), the infrared spectrum of the sample in the range of $4000-400 \mathrm{~cm}^{-1}$ was collected using $\mathrm{KBr}$ pellet. The ultraviolet-visible spectrum (UV-Vis) was recorded on an UV-Vis-NIR spectrophotometer (Agilent,
Palo Alto, USA), using ethanol as the background and scanning range of $200-500 \mathrm{~nm}$. Thermogravimetric analysis (TG) was performed on STA449 thermogravimetric analyzer (Netzsch, Germany). About $10 \mathrm{mg}$ of sample were subjected to thermogravimetric analysis at $50 \mathrm{~mL} \mathrm{~min}{ }^{-1}$

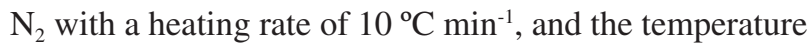
range was $25-800{ }^{\circ} \mathrm{C}$. Scanning electron microscopy images (SEM) were obtained with a JSM6380LV (JEOL, Tokyo, Japan) instrument to observe the morphology of the prepared samples.

\section{Catalytic nitration of benzene}

The catalytic nitration of benzene was carried out in a $100 \mathrm{~mL}$ three-mouth bottle. In a typical experiment, a certain amount of catalyst, benzene and nitric acid were placed in a bottle successively. The reaction mixture was then placed in an oil bath and heated to the desired temperature under continuous stirring. After the reaction, the mixture was cooled to room temperature. Solid catalyst was separated from the product by filtration. The organic phase was analyzed by gas chromatography-mass spectrometry (GC-MS, HP-5MS capillary column) to determine the product composition and nitrobenzene yield. The detailed GC-MS analysis conditions were: the temperature was kept at $50^{\circ} \mathrm{C}$ for $1 \mathrm{~min}$, then the temperature was increased to $180{ }^{\circ} \mathrm{C}$ at a rate of $15^{\circ} \mathrm{C} \mathrm{min}-1$, finally held at $180{ }^{\circ} \mathrm{C}$ for $1 \mathrm{~min}$. Two peaks appeared at retention time 2.1 and $6.6 \mathrm{~min}$, which correspond to benzene and nitrobenzene, respectively.

\section{Results and Discussion}

\section{Catalysts characterization}

The XRD patterns of HPMV, QA and the prepared QA-HPMV are shown in Figure 1. HPMV has obvious characteristic diffraction peaks at $2 \theta=7.2,9.1,20.2$ and $25-30^{\circ}$, which was consistent with the spectrogram of $\mathrm{H}_{4} \mathrm{PMo}_{11} \mathrm{VO}_{40}$ with Keggin structure reported in the literature. ${ }^{22-24}$ QA exhibits a highly crystalline structure and these diffraction peaks mainly appeared in the range of 5-30 $0^{\circ}$ QA-HPMV shows some diffraction peaks at $2 \theta=5-10,18.4,21.2$ and $27.5^{\circ}$. It could be found that the XRD pattern of QA-HPMV was not a simple superposition of these diffraction peaks of HPMV and QA, indicating that QA-HPMV was not the mixture of QA and HPMV, but a new crystalline phase. These peaks of QA-HPMV and HPMV are all mainly presented in the four similar regions, so it is speculated that QA-HPMV possesses the same symmetry as parent HPMV. ${ }^{25}$ 
The XRD patterns of NA-HPMV and DTAB-HPMV are also shown in Figure 1. It indicated that new crystalline phase is formed after the reaction of HPMV and QA or DTAB. However, compared with QA-HPMV and NAHPMV, DTAB-HPMV has poor crystallinity.

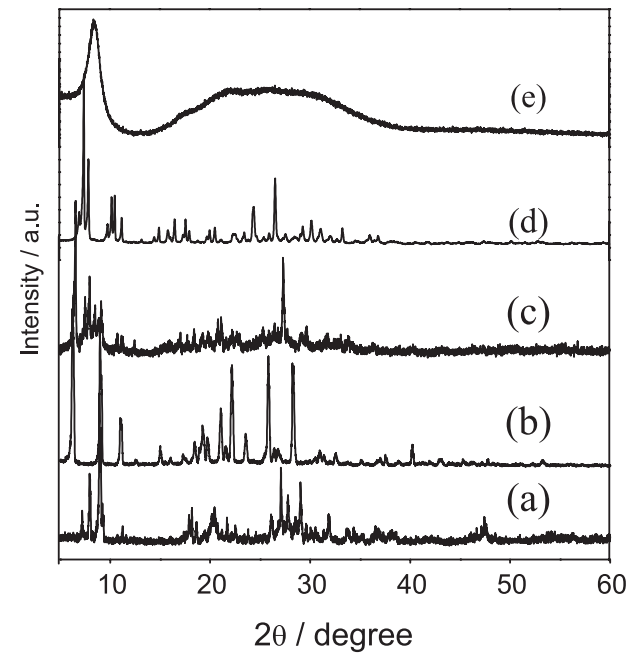

Figure 1. XRD patterns of (a) HPMV, (b) QA, (c) QA-HPMV, (d) NA-HPMV, (e) DTAB-HPMV.

The Fourier transform infrared spectroscopy (FTIR) spectra of HPMV, QA and QA-HPMV are shown in Figure 2. In the region from 1100 to $800 \mathrm{~cm}^{-1}$, HPMV clearly shows four characteristic absorption bands of Keggin structure at $1062,962,867,786 \mathrm{~cm}^{-1}$. These bands could be attributed to $v\left(\mathrm{P}=\mathrm{O}_{\mathrm{a}}\right), v\left(\mathrm{Mo}=\mathrm{O}_{\mathrm{t}}\right), v\left(\mathrm{Mo}-\mathrm{O}_{\mathrm{b}}-\mathrm{Mo}\right)$ and $v\left(\mathrm{Mo}-\mathrm{O}_{\mathrm{c}}-\mathrm{Mo}\right)$, respectively $\left(\mathrm{O}_{\mathrm{a}}\right.$ : tetrahedron oxygen; $\mathrm{O}_{\mathrm{t}}$ : terminal oxygen, $\mathrm{O}_{\mathrm{b}}$ : bridged oxygen of two octahedral sharing a corner and $\mathrm{O}_{\mathrm{c}}$ : bridged oxygen sharing an edge). ${ }^{22,26,27}$ For QA-HPMV, also, four characterisitic bands appear at 1047, 956, 861 and $781 \mathrm{~cm}^{-1}$, indicating that QA-HPMV retained the Keggin structure of HPMV. The red shift of the corresponding peak maybe due to the interaction between HMPV and QA. In the region from 1700 to $1100 \mathrm{~cm}^{-1}$, some characteristic bands related to QA were observed in the spectra of QA-HPMV. ${ }^{28}$ Combined with the result of XRD, it obviously revealed the successful combination of QA and HPMV. ${ }^{29}$

The UV-Vis spectra of HPMV, QA and QA-HPMV are shown in Figure 3. The UV-Vis absorption spectrum is generated by valence electron transition, and the UV-visible absorption of polyoxometallate can occur at $200-400 \mathrm{~nm} \cdot{ }^{30,31}$ The UV-Vis spectrum of HPMV shows absorption peaks at 221 and $308 \mathrm{~nm}$, which is caused by the charge transfer $\mathrm{O} \rightarrow \mathrm{Mo}$ and $\mathrm{O} \rightarrow \mathrm{V}$ in $\left[\mathrm{PMo}_{11} \mathrm{VO}_{40}\right]^{4-}$ anion, respectively. ${ }^{14,23}$ QA shows three adsorption peaks at 204, 232 and $287 \mathrm{~nm}$, which can be attributed to the $\pi \rightarrow \pi^{*}$ electron transition of quinoline ring. For the prepared QA-HPMV, these two

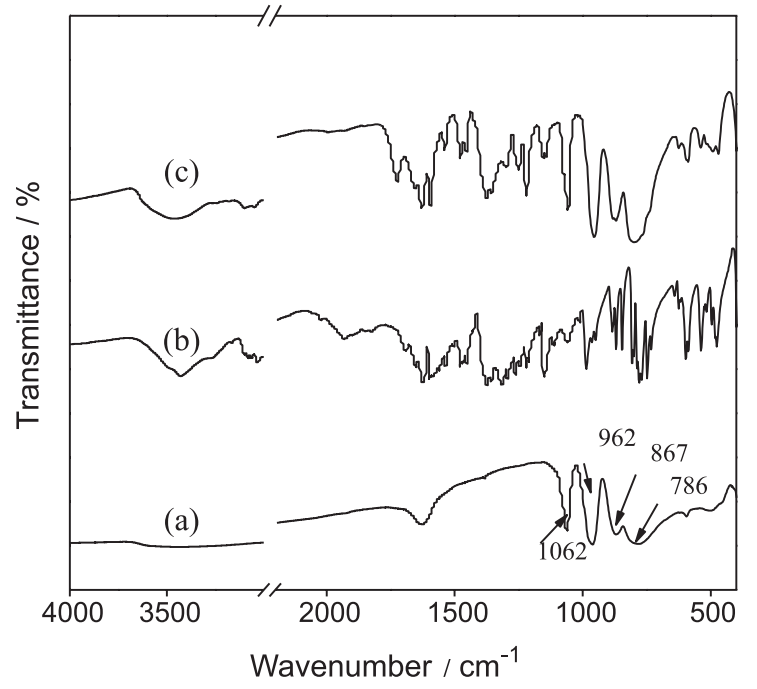

Figure 2. FTIR (KBr) spectra of (a) HPMV, (b) QA, (c) QA-HPMV.

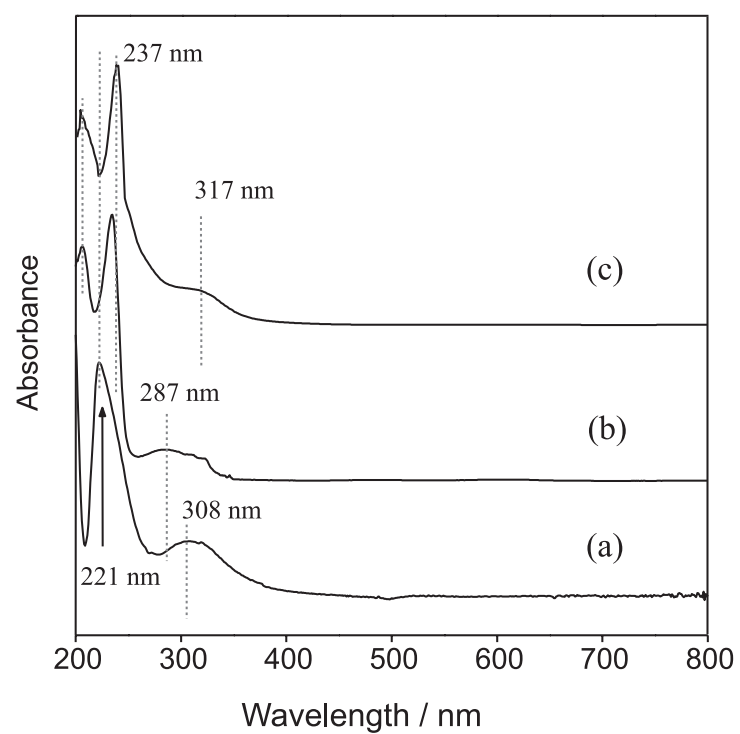

Figure 3. UV-Vis spectrum of (a) HPMV, (b) QA and (c) QA-HPMV.

absorption peaks related to the charge transfer $\mathrm{O} \rightarrow \mathrm{Mo}$ and $\mathrm{O} \rightarrow \mathrm{V}$ all move to higher wavelength and appear at 237 or $317 \mathrm{~nm}$, which may be due to the electron interaction between QA and the Keggin unit of HPMV.

Thermal gravimetric analysis was used to study the thermal stability of QA-HPMV and the result is shown in Figure 4A. For comparison, the TG curves of HPMV and QA are also listed. HPMV has a weight loses about $9.5 \%$ at $110^{\circ} \mathrm{C}$, owing to the removal of free water on the surface of the sample. The subsequent slow weight loss process of HPMV is due to the thermal decomposition of $\left[\mathrm{PMo}_{11} \mathrm{VO}_{40}\right]^{4-}$ into oxides. ${ }^{27} \mathrm{QA}$ shows a sharp degradation with about $50 \%$ weight loss between 140 and $210^{\circ} \mathrm{C}$. QA-HPMV shows good stability at less than $200{ }^{\circ} \mathrm{C}$ and only has a little weight loss, especially the sharp degradation similar to QA was not found. These results 

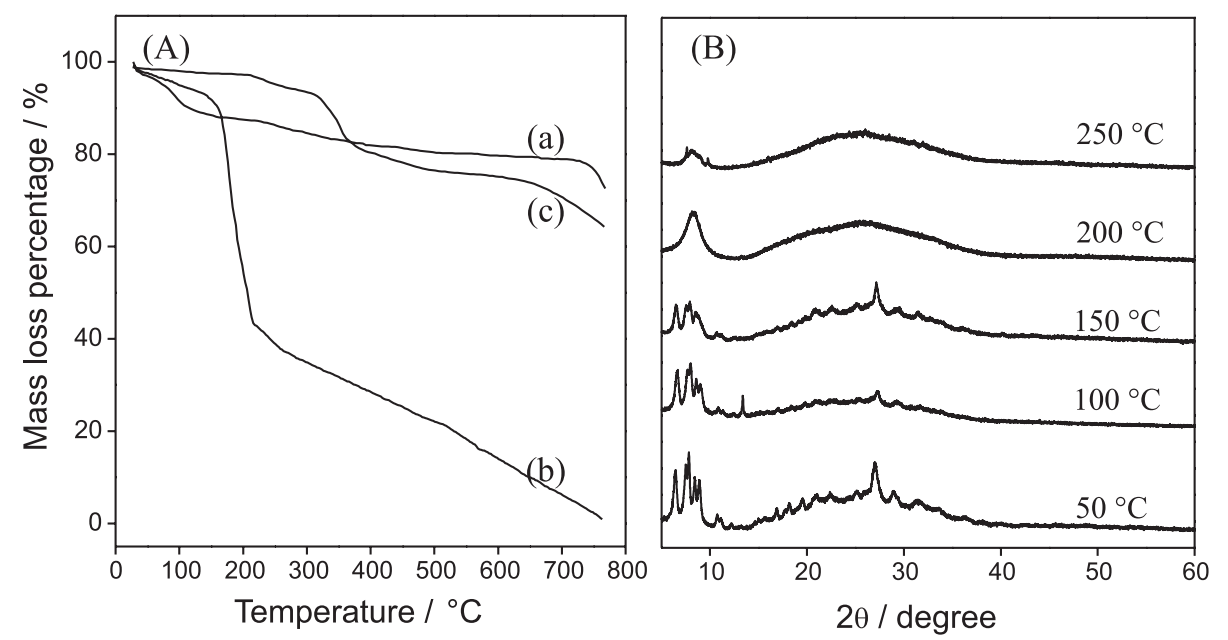

Figure 4. (A) TG patterns of (a) HPMV, (b) QA, (c) QA-HPMV and (B) XRD patterns of QA-HPMV catalyst at different temperatures.

further confirmed the combination of QA and HPMV. However, when the temperature is higher than $200^{\circ} \mathrm{C}$, QA-HPMV begins to have significant weight loss, which may be caused by the loss of organic ligand QA in the decomposition of QA-HPMV, and the later weightlessness may be caused by the decomposition of HPMV. To further verify the stability of QA-HPMV, XRD analysis was carried out after heating at different temperatures for $30 \mathrm{~min}$ and the result is shown in Figure 4B. It can be seen that the XRD pattern of the sample treated at $150{ }^{\circ} \mathrm{C}$ has no obvious change except that the intensity of the corresponding peaks decreases. However, after treated at $200{ }^{\circ} \mathrm{C}$, the XRD pattern is completely different, indicating that the phase has changed. Therefore, combined with XRD and TG results, QA-HPMV has good thermal stability below $200{ }^{\circ} \mathrm{C}$.

The surface morphology of HPMV, QA and QA-HPMV was studied by SEM and the typical micrographs are shown in Figure 5. HPMV has no regular morphology, and it is clearly observed that the particles are large in size and agglomerate obviously. On the contrary, QA shows a regular strip shape. QA-HPMV is not a lumpy with obvious agglomeration phenomenon, nor a single regular strip, which indicates that their original morphology will change after the combination of QA and HPMV. When the magnification is small, QA-HPMV is a rose-like shape with petals stacked on top of each other. After magnification, it can be found that QA-HPMV has irregular flakes, some of which are similar to petals.

\section{Catalytic activity assessment}

In order to investigate the catalytic performance of these three samples, including QA-HPMV, NA-HPMV and DTAB-HPMV, the nitration of benzene was carried out under the condition that the catalyst dosage was $1 \%$ of

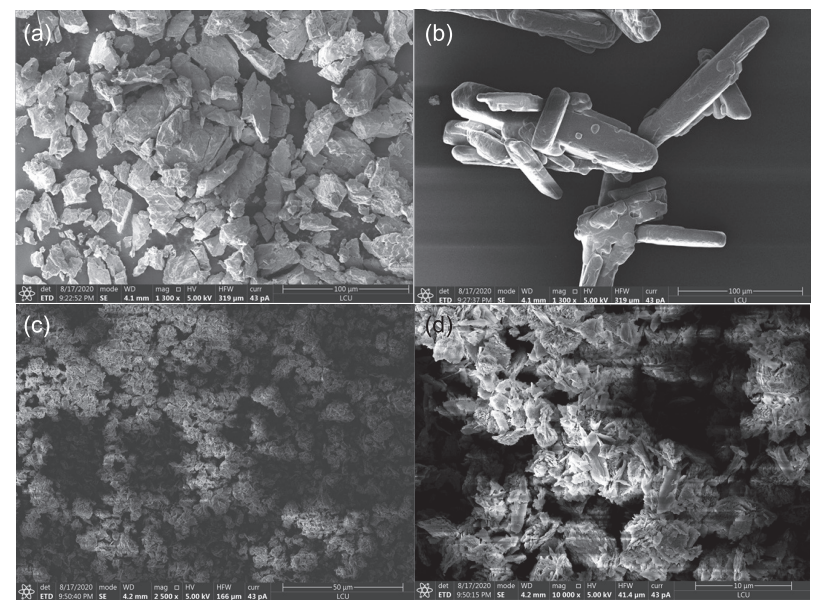

Figure 5. SEM images of (a) HPMV, (b) QA, (c) QA-HPMV (2500x) and (d) QA-HPMV (10000x).

the benzene mass, the molar ratio of nitric acid to benzene was $1.1: 1$, the reaction time was $6 \mathrm{~h}$ and the reaction temperature was $60{ }^{\circ} \mathrm{C}$. The reaction results are shown in Table 1. The nitrobenzene could be formed as the only product from benzene with nitric acid in the absence of any catalyst, however, the yield was only $27.8 \%$. Soluble HPMV and NA-HPMV exhibit good activity, the yields of nitrobenzene were 55.8 and $60.3 \%$, respectively. However, the complete dissolution results in homogeneous reaction bringing complicated process for catalyst recycling. QAHPMV and DTAB-HPMV are insoluble in the reaction system, they can be recycled through simple filtration and the recovery rate is 94 and $96 \%$, respectively. This is conducive to sustainable development. Among them, QA-HPMV exhibits the best catalytic activity, the yield of nitrobenzene was $70.4 \%$ under the same conditions.

The reaction performance of QA-HPMV was optimized by systematically exploring the influence of various reaction parameters including the reaction temperature, 
Table 1. Nitration of benzene catalytic by different catalysts

\begin{tabular}{lcc}
\hline Catalyst & Catalyst solubility & Yield / \% \\
\hline None & & 27.8 \\
HPMV & soluble & 55.8 \\
QA-HPMV & insoluble & 70.4 \\
NA-HPMV & soluble & 60.3 \\
DTAB-HPMV & insoluble & 58.9 \\
\hline
\end{tabular}

HPMV: molybdovanadophosphoric heteropolyacid QA: quinoline2-formic acid; NA: nicotinic acid; DTAB: dodecyl trimethyl ammonium bromide.

reaction time, nitration agent dosage, catalyst dosage, and the results are shown in Figure 6.

When the molar ratio of nitric acid to benzene was $1.1: 1$, the amount of catalyst was $2 \%$ of the benzene mass, and the reaction time was $6 \mathrm{~h}$, the influence of reaction temperature on nitration was investigated, as shown in Figure 6a. The yield of nitrobenzene rapidly increased from 26.7 to $75.2 \%$ with the raised reaction temperature from 25 to $60^{\circ} \mathrm{C}$. However, from 60 to $75^{\circ} \mathrm{C}$, the yield increased slowly, only by $0.4 \%$, which may be due to the increase of temperature will accelerate the decomposition of nitric acid into nitrogen dioxide and then lead to the decrease of nitric acid concentration in reaction system. Then, the appropriate reaction temperature was set as $60{ }^{\circ} \mathrm{C}$ for the following experiments.

The effect of reaction time on nitration was investigated at $60^{\circ} \mathrm{C}$ with the molar ratio of nitric acid to benzene was 1.1:1, and the amount of catalyst was $2 \%$ of the benzene mass. It can be seen from Figure $6 \mathrm{~b}$ that prolonging the reaction time is beneficial to the reaction. The yield of nitrobenzene increased from 40.8 to $75.2 \%$ when the reaction time was increased from 1 to $6 \mathrm{~h}$. When the reaction time was increased to $8 \mathrm{~h}$, the yield of nitrobenzene did not change significantly. Therefore, the optimal reaction time was $6 \mathrm{~h}$.

In this nitration process, generated water will dilute nitric acid and weaken its nitration ability, so the amount of nitric acid should be excessive. Figure $6 \mathrm{c}$ shows the effect of molar ratio of nitric acid to benzene on the nitration reaction, when the reaction temperature was $60^{\circ} \mathrm{C}$, reaction time was $6 \mathrm{~h}$ and catalyst dosage was $2 \%$ of the benzene mass. The yield of nitrobenzene increased from 75.2 to $78.8 \%$ with increasing the molar ratio of nitric acid to benzene from $1.1: 1$ to $2: 1$, and the yield reached $79.4 \%$ when the molar ratio of nitric acid to benzene was $2.7: 1$.
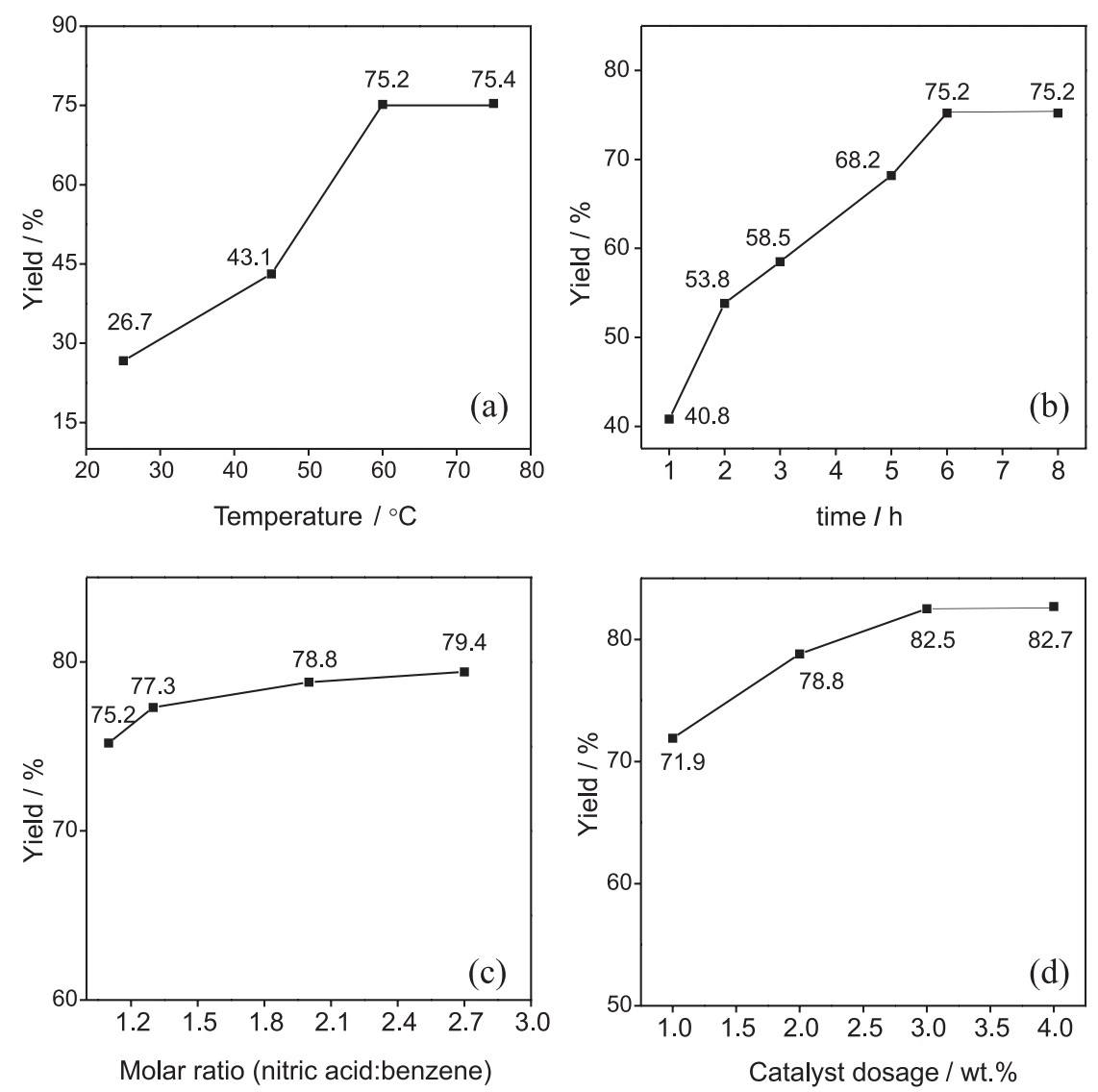

Figure 6. Effects of reaction conditions on the nitration reaction over QA-HPMV catalyst (a) reaction temperature ( $2 \%$ catalyst amount, nitric acid/benzene molar of $1.1,6 \mathrm{~h})$; (b) reaction time ( $2 \%$ catalyst amount, nitric acid/benzene molar of $\left.1.1,60{ }^{\circ} \mathrm{C}\right) ;(\mathrm{c})$ amount of nitric acid $\left(2 \%\right.$ catalyst amount, $60{ }^{\circ} \mathrm{C}$, $6 \mathrm{~h})$; (d) catalyst content (nitric acid/benzene molar of $2,60^{\circ} \mathrm{C}, 6 \mathrm{~h}$ ). 
Despite some increase, a small amount of by-product dinitrobenzene was also detected. Therefore, in order to obtain a high yield and easy purification, the molar ratio of nitric acid to benzene was selected as 2:1.

Figure $6 \mathrm{~d}$ shows the effect of QA-HPMV dosage on the nitration of benzene with nitric acid. Generally speaking, the appropriate increase of catalyst dosage will be beneficial to the reaction, which is also verified by the result of nitration of benzene catalytic by QA-HPMV. The yield of nitrobenzene increased from 71.9 to $82.5 \%$ when the amount of catalyst increased from 1 to $3 \%$ of the benzene mass. When the amount of catalyst is $4 \%$ of benzene by weight, the yield of nitrobenzene is almost unchanged, which may be due to the presence of excessive catalyst in the reaction system, leading to a decrease in the contact opportunity of benzene and nitric acid. ${ }^{25}$

Therefore, based on the above results, these suitable reaction conditions were obtained: the reaction temperature was $60{ }^{\circ} \mathrm{C}$, reaction time was $6 \mathrm{~h}$, the molar ratio of nitric acid to benzene was 2:1 and the amount of QA-HPMV catalyst was $3 \%$ of the benzene mass. Under these reaction conditions, a relatively high yield of $82.5 \%$ was obtained.

The reusability of QA-HPMV catalyst

After reaction, the QA-HPMV was conveniently recovered by filtration and reused for the next run to evaluate the catalytic reusability. Figure 7 displays the result of a five run test under these optimal reaction conditions. It can be seen that the QA-HPMV catalyst shows good reusability in the nitration reaction. After five times of use, the yield of nitrobenzene is still above $80 \%$, there is a slightly decreased by $1.9 \%$ for the final run compared with that of the first run.

After reuse, the catalyst was collected to dry at $80^{\circ} \mathrm{C}$, and then was characterized by FTIR. As shown in Figure 8,

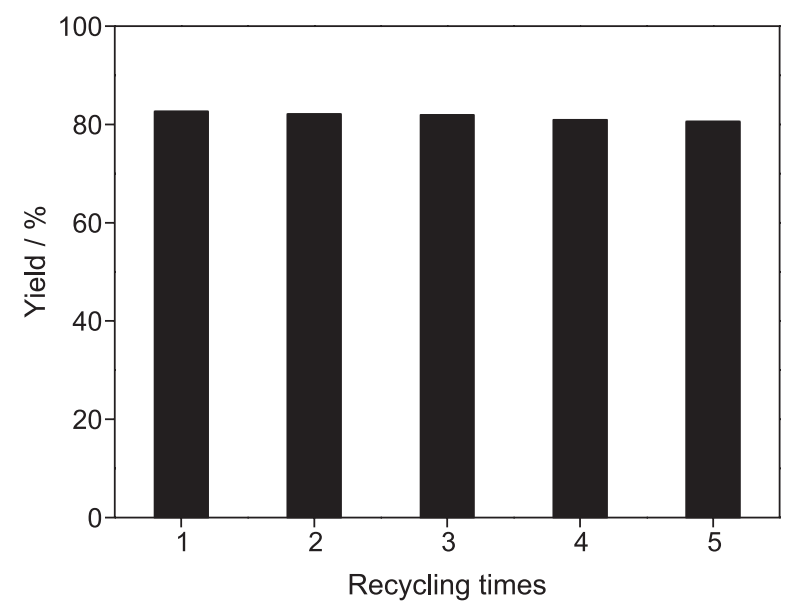

Figure 7. The reusability of QA-HPMV. the recycled catalyst has a similar infrared spectrum to fresh QA-HPMV, indicating that the Keggin unit structure was well maintained during the reaction. These results show that QA-HPMV could be a promising new type of heterogeneous catalyst for the nitration of benzene.

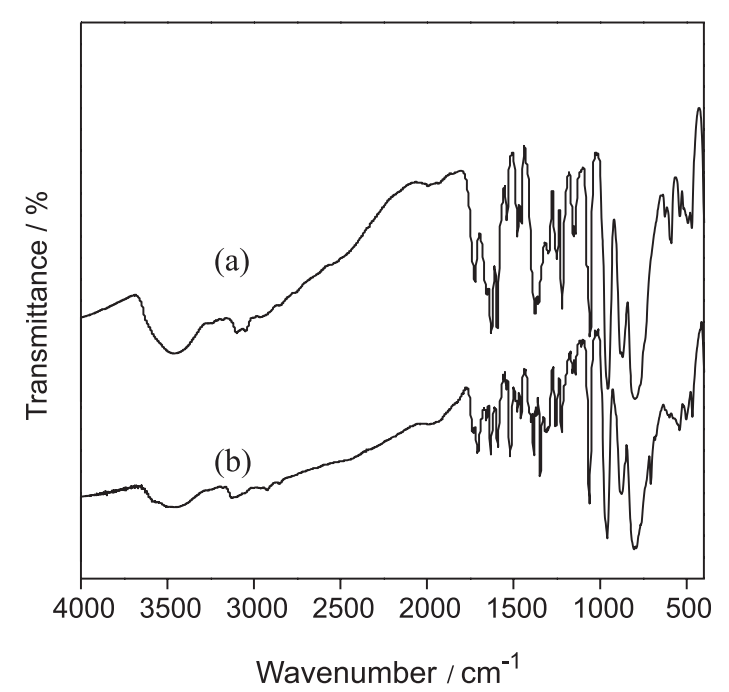

Figure 8. FTIR (KBr) spectra of (a) QA-HPMV and (b) used QA-HPMV.

\section{Conclusions}

In this work, a novel organic salt of QA-HPMV with Keggin structure was prepared through the reaction of molybdovanadophosphoric heteropolyacid and quinoline2 -formic acid in aqueous solution. This salt displayed an excellent catalytic performance for the nitration of benzene with nitric acid as nitration agent, including high nitrobenzene yield and good reusability. Under the optimized conditions, a relative high yield of $82.5 \%$ can be obtained. After reaction, the catalyst could be easily recycled by filtration, and its fine structure including the Keggin unit structure was not damaged during the reaction. Therefore, as a substitute for sulfuric acid, QA-HPMV may be an excellent environmentally friendly solid catalyst for nitration of benzene.

\section{Acknowledgments}

The authors are grateful for the support of the Shandong Provincial Natural Science Foundation (ZR2017MB060), the National Natural Science Foundation of China (21101086) and the Foundation of Liaocheng University (318011406).

\section{References}

1. Sato, H.; Hirose, K.; Appl. Catal., A 1998, 174, 77. 
2. Zhou, S.; You, K.; Gao, H.; Deng, R.; Zhao, F.; Liu, P.; Ai, Q.; Luo, H.; Mol. Catal. 2017, 433, 91.

3. Gong, S.; Liu, L.; Cui, Q.; Ding, J.; J. Hazard. Mater. 2010, 178, 404.

4. Ono, N.; The Nitro Group in Organic Synthesis; Wiley-VCH: New York, 2001.

5. Nishiwaki, N.; Molecules 2020, 25, 3680.

6. Cheng, G.; Duan, X.; Qi, X.; Lu, C.; Catal. Commun. 2008, 10, 201.

7. Quadros, P. A.; Oliveira, N. M. C.; Baptista, C. M. S. G.; Chem. Eng. Sci. 2004, 59, 5449.

8. Yuan, Y.; Nie, J.; Zhang, Z.; Wang, S. H.; Appl. Catal., A 2005, 259,170 .

9. Qiao, K.; Hagiwara, H.; Yokoyama, C.; J. Mol. Catal. A: Chem. 2006, 246, 65.

10. Choudary, B. M.; Sateesh, M.; Lakshwi Kantam, M.; Koteswara Rao, K.; Ram Prased, K. V.; Raghavan, K. V.; Sarma, J. A. R. P.; Chem. Commun. 2000, 31, 25.

11. Ganjala, V. S. P.; Neeli, C. K. P.; Pramod, C. V.; Khagga, M.; Rao, K. S. R.; Burri, D. R.; Catal. Commun. 2014, 49, 82.

12. Bertea, L. E.; Kouwenhoven, H. W.; Prins, R.; Stud. Surf. Sci. Catal. 1994, 84, 1973.

13. Brei, V. V.; Prudius, S. V.; Melezhyk, O. V.; Appl. Catal., A $\mathbf{2 0 0 3}, 239,11$.

14. Li, L.; Xu, Z.; Liu, F.; Shao, Y.; Wang, J.; Wan, H.; Zheng, S.; J. Photochem. Photobiol., A 2010, 212, 113.

15. Palermo, V.; Sathicq, A.; Constantieux, T.; Rodriguez, J.; Vazquez, P.; Romanelli, G.; Catal. Lett. 2015, 145, 1022.

16. Palermo, V.; Sathicq, A.; Constantieux, T.; Rodriguez, J.; Vazquez, P.; Romanelli, G.; Catal. Lett. 2016, 146, 1634.

17. Heravi, M. M.; Benmorad, T.; Bakhtiari, K.; Bamoharrm, F. F.; Oskooie, H. H.; J. Mol. Catal. A: Chem. 2007, 264, 318.
18. Villabrille, P.; Romanelli, G.; Vázquez, P.; Cáceres, C.; Appl. Catal., A 2004, 270, 101.

19. Romanelli, G.; Sathicq, A.; Vázquez, P.; Villa, A.; Alarcón, E.; Grajales, E.; Cubillos, J.; J. Mol. Catal. A: Chem. 2015, 398, 11.

20. You, K.; Deng, R.; Jian, J.; Liu, P.; RSC Adv. 2015, 5, 73083.

21. Tsigdinos, G. A.; Hallada, C. J.; Inorg. Chem. 1968, 7, 437.

22. Yin, Y.; Wang, H.; Yang, Y.; Fan, R.; Dong, G.; Wei, L.; Chin. Chem. Lett. 2016, 27, 613.

23. Kala Raj, N. K.; Ramaswamy, A.V.; J. Mol. Catal. A: Chem. 2005, 227, 37.

24. Yang, W.; Wang, L.; Wang, Q.; Chin. J. Inorg. Chem. 2013, 29 , 295.

25. Zhou, X.; Li, Z.; Zhang, C.; Gao, X.; Dai, Y.; Wang, G.; J. Mol. Catal. A: Chem. 2016, 417, 71.

26. Neumann, R.; Vega, M.; J. Mol. Catal. 1993, 84, 93.

27. Jing, L.; Zhang, F.; Zhong, Y.; Zhu, W.; Chin. J. Chem. Eng. 2014, 22, 1220.

28. Khreis, O. M.; Curry, R. J.; Somerton, M.; Gillin, W.; J. Appl. Phys. 2000, 88, 777.

29. de Oliveira, A. N.; de Lima, M. A. B.; Pires, L. H. O.; da Silva, M. R.; da Luz, P. T. S.; Angélica, R. S.; da Rocha Filho, G. N.; da Costa, C. E. F.; Luque, R.; do Nascimento, L. A. S.; Materials 2019, 12, 1431.

30. Wang, H.; Chen, Y.; You, Z.; Zhou, M.; Zhang, N.; Sun, Y.; Chin. Chem. Lett. 2015, 26, 187.

31. Liu, L.; Luan, Q.; Lu, J.; Lv, D.; RSC Adv. 2018, 8, 26180.

Submitted: December 16, 2020

Published online: March 3, 2021 